\title{
Polysiloxane/Gadolinium Chelates-based Nanoparticles
}

\author{
National Cancer Institute
}

\section{Source}

National Cancer Institute. Polysiloxane/Gadolinium Chelates-based Nanoparticles. NCI

Thesaurus. Code C129651.

A nanoparticle formulation composed of a polysiloxane-based inorganic matrix bound to cyclic chelates composed of the chelating agent DOT A $(1,4,7,10$-tetraazacyclododecane-1-glutaric anhydride-4,7,10-triacetic acid) covalently bound to the paramagnetic contrast enhancer gadolinium (Gd), a high-Z material, with potential use in enhancing diagnostic images upon magnetic resonance imaging (MRI) and potential radiosensitizing activity. Upon intravenous administration of polysiloxane matrixgadolinium chelates-based nanoparticles, the nanoparticles extravasate to tumor sites due to the unique, hyperpermeable environment of the tumor vasculature. In addition, the small nanoparticles allow for deep penetration into the tumor, where they subsequently accumulate. Upon placement in a magnetic field, this agent produces a large magnetic moment and creates a large local magnetic field, which can enhance the relaxation rate of nearby protons. This change in proton relaxation dynamics increases the MRI signal intensity of tissues in which this agent has accumulated; therefore, contrast and visualization of those tissues are enhanced compared to unenhanced MRI. The ultra-small nanoparticles, less than $5 \mathrm{~nm}$ in diameter, allow for rapid renal clearance and reduced toxicity. Due to their magnetic properties, these nanoparticles make tumor cells more sensitive to radiation therapy. 